\title{
Optimal placement of DSTATCOM in distribution network based on load flow and voltage stability indices studies
}

\begin{abstract}
The power utilities are experiencing a new challenge as the demand for electrical power is increasing on a regular basis. This forced the existing networks operates closed to its stability limits. The ability of a power system to remain acceptable magnitude voltages at all bus under both normal and contingency conditions, known as voltage stability is the major concern for both transmission and distribution network to ensure that a secure and reliable electrical power is able to transmit from generation to load side. This can be mitigated by integrating Custom Power (CP) device on the existing transmission system. However, the location of $\mathrm{CP}$ device is significantly important to ensure adequate investment of $\mathrm{CP}$ device in networks that will enhance the voltage stability margin, reduce power loss and improve voltage profile. In this paper, the optimal location of Distribution Static Synchronous Compensator (DSTATCOM) is introduced by analyzing results obtained from two studies; load flow and voltage stability indices. The studies were examined using the modified IEEE 30 bus system which is modelled and tested using DigSILENT PowerFactory 16 as simulation tools.
\end{abstract}

Keyword: Optimal placement; Custom power devices; DSTATCOM; Voltage stabiliy indices; Load flow 